\title{
Public Perception of Facial Fillers
}

\author{
Houmehr Hojjat, MD ${ }^{1}$ Richard Raad, BS ${ }^{1}$ Jordyn Lucas, MD ${ }^{1}$ Mohammad Mir, BS ${ }^{1}$ \\ Brendan C. Smith, MS ${ }^{2}$ Michael A. Carron, MD ${ }^{1,3}$ Giancarlo Zuliani, MD ${ }^{1,4}$
}

${ }^{1}$ Department of Otolaryngology - Head and Neck Surgery, Wayne

Address for correspondence Brendan Smith, MS, Department of State University School of Medicine, Detroit, Michigan

2 Department of Otolaryngology - Head and Neck Surgery, Rutgers Otolaryngology - Head and Neck Surgery, Rutgers New Jersey Medica School, 90 Bergen Street, Suite 8100, Newark, NJ 07103 New Jersey Medical School, Newark, New Jersey

${ }^{3}$ Division of Facial Plastic and Reconstructive Surgery, Wayne State (e-mail: smithbc@njms.rutgers.edu).

University School of Medicine, Detroit, Michigan

${ }^{4}$ Zuliani Facial Aesthetics, Bloomfield Hills, Michigan

Facial Plast Surg 2019;35:204-209.

\begin{abstract}
Keywords

- facial fillers

- demographics

- public perception

- Google News

There has been widespread coverage of cosmetic procedures, particularly facial fillers, as minimally invasive options for facial rejuvenation. The authors' objective was to characterize news media's coverage of facial fillers and its role in shaping public perception of these products over the past decade. Public view plays a significant role in policymaking, assisting patient communication, and addressing preconceived notions. Google News was searched for online news coverage related to "facial fillers" from 2008 to 2017. News articles from various sources were reviewed and analyzed with multiple objectives including complications listed, advantages, disadvantages, physician specialties, overall theme of the articles as positive, negative, or neutral and other parametrics. A chi-square test was used for statistical analysis. Of 426 articles meeting inclusion criteria, international news (20.4\%), tabloids (18.8\%), online health sites (18.3\%), and national news (16.9\%) were represented. Of articles containing adequate information, coverage was $44.7 \%$ positive, $29.9 \%$ neutral, and $26.1 \%$ negative with no significant change from 2008 to 2017 . In addition, $46.0 \%$ of papers discussed complications including vascular injury and blindness associated with fillers, with significant increase by $2017(p<0.05)$. Facial fillers media coverage was overall positive, with broad coverage at both national and international levels. Complications were discussed often, and although vascular injury and vision damage are rare complications of fillers, they were frequently cited, potentially leading the public to believe they occur frequently. There were also significant concerns in the news media about greater regulation of products used as facial fillers, and practitioners administrating them. Altogether, these findings provide a comprehensive overview of patient perception and expectations of an increasingly popular and expanding cosmetic procedure.
\end{abstract}

Injectable facial fillers have become a popular option for facial rejuvenation in recent years, with over 2 million dermal filler treatments performed in the United States in 2016. ${ }^{1}$ Facial fillers are used to restore facial volume, reverse aging, and enhance the shape and contours of the face. $^{2}$ Fillers first emerged in the early 1980 s and have evolved from the use of substances such as bovine collagen, which was associated with the possibility of hypersensitiv- ity events and suboptimal results, to the incorporation of fillers with greater biocompatibility and safety profile. ${ }^{3}$ There are currently various commercially available filler options including hyaluronic acid (HA), calcium hydroxylapatite, and Poly-L-lactic acid products. ${ }^{2}$ HA products are the most widely used fillers. Their biochemical properties make them the ideal structural compound with a low potential for allergic or immunogenic reaction. ${ }^{3}$ Another benefit is published online March 8, 2019
Issue Theme Aesthetic Treatment of the Jawline and Perioral Area; Guest Editor: S. Randolph Waldman, MD
Copyright $\odot 2019$ by Thieme Medical Publishers, Inc., 333 Seventh Avenue, New York, NY 10001, USA. Tel: +1(212) 584-4662.
DOI https://doi.org/ 10.1055/s-0039-1681071. ISSN 0736-6825. 
the ability to dissolve and reverse HA-based products using hyaluronidase. ${ }^{4}$

In the past, facial fillers had limited use due to a combination of factors including longevity of results, the social stigma of having injections performed, and hypersensitivity reactions. This has changed drastically since the advent of biocompatible fillers. Since 1997, facial filler use in the United States has increased $356 \%{ }^{5}$ Another reason for the exponential increase has been the popularization of fillers and botulinum toxin in social media and news coverage, both locally and internationally. For instance, the average interest level in facial fillers increased by $30 \%$ after Kylie Jenner announced using Juvederm (Allergan) lip injections. ${ }^{6}$

It is common practice for patients to research medical information online. In a study conducted by the Pew Internet and American Life Project, 68\% of respondents stated that recently searched information online greatly impacted their health decisions, as well as the health decisions of their loved ones. ${ }^{7}$ Therefore, both the quantity and quality of news media that people are exposed to can play a significant role in their perceptions, expectations, and ultimately their medical decision-making. ${ }^{8}$ This study investigates how the evolving nature of news media has portrayed facial fillers and analyzes changes in trends and coverage over the past decade.

\section{Methods}

Google News is an online search engine that aggregates news articles from thousands of online news sources and blogs. ${ }^{9}$ This search engine uses an internal algorithm together with the user's search terms to yield relevant news articles from a wide variety of sources. ${ }^{9}$ Search results may be limited to a specified time period, sorted by relevance to the search terms or by date, and filtered to include all news articles or limited to blog articles. Google News has shown its value in news coverage analyses encompassing a variety of other medical topics to date. ${ }^{8,10,11}$

The news search was performed in June 2018 using the search terms "facial fillers." It encompassed a 10-year period, from January of 2008 through January of 2018. It was divided into 10 consecutive 1 -year periods, with the first period spanning from 01/01/2008 until 01/01/2009 and the last period spanning from $01 / 01 / 2017$ until $01 / 01 / 2018$. The personalization feature for Google Search was disabled for each reviewer to ensure consistent results. English-written articles from both American and international news sources were included and categorized accordingly. Exclusion criteria included duplicate articles, those requiring login credentials to view, and videos and those unrelated to the search terms listed above. The search was limited to the first 50 articles meeting inclusion criteria in each 1 -year period. In total, 426 articles met inclusion criteria for this analysis, and three authors (H.H., R.R., and M.M.) independently reviewed each article for accuracy and consistency.

\section{Data Evaluation}

Parameters utilized to evaluate each article included article source, overall portrayal of facial fillers (positive, negative, or neutral), mention of specific brands versus generic terms, advantages/disadvantages compared with alternatives, complications, pricing, celebrity-related articles, gender, before/ after pictures and testimonials, whether mechanism of action was listed, physician specialty, and geographic location of the articles. All article sources were divided into the following categories in the final analysis: business news outlet (e.g., Forbes magazine), local news outlet (e.g., The Detroit News), national news outlet (e.g., USA Today), advertisement, blog, online health/medical source (e.g., WebMD), and tabloids (e.g., People Magazine).

Given that interpretation of each article's overall portrayal of facial fillers (as positive, negative, or neutral) is inherently subjective, several efforts were made to objectify this process. Articles categorized as providing positive portrayal of facial fillers listed more advantages than disadvantages compared with alternatives (e.g., plastic surgery), included positive patient testimonials, and/or a greater number of supportive comments from physicians than unsupportive ones. On the other hand, articles categorized as portraying facial fillers negatively listed more disadvantages or complications of facial fillers than advantages, included negative patient testimonials, and/or more unsupportive comments from physicians. Neutral articles were categorized as such because they provided an overall balanced perspective of facial fillers, with the differential between advantages and disadvantages equal to or less than two. Three reviewers independently rated the articles in this manner (H.H., R.R., and M.M.).

\section{Statistical Analysis}

A chi-square test was used for comparison between media sources in the nature of their coverage and reporting of complications, disadvantages, and outcomes. Threshold for significance was set at $p<0.05$, and SPSS version 20 (IBM Inc.) was used for statistical calculations.

\section{Results}

The number of articles per year more than doubled from 2008 to 2009 then remained steady throughout the rest of the study period (-Fig. 1) Of the 426 articles meeting inclusion criteria, international coverage of facial fillers was the highest, representing $20.4 \%$ of total news coverage with $90.0 \%$ originating from Britain. After international coverage, local tabloids were the second highest in terms of representation (18.8\%), followed by US national news outlets (16.9\%) and online medical/health sources at $18.3 \%$. Of articles originating from the United States, $37.2 \%$ were from the West Coast, 36.1\% from the East Coast, 16.0\% from the South, and $10.6 \%$ from the Midwest.

Upon analyzing demographics, $47.7 \%$ of the papers specifically mentioned gender, with $77 \%$ referencing females. Celebrity-related articles also comprised $27 \%$ of the articles. However, only $23.9 \%$ of articles included a testimonial from a patient with $9.6 \%$ including before/after pictures.

After examining general themes among the news articles, the most common were "increased demand" and "innovation in technology" (14.2\% each). Innovations included introduction of new fillers, novel application of fillers, and adjuncts 


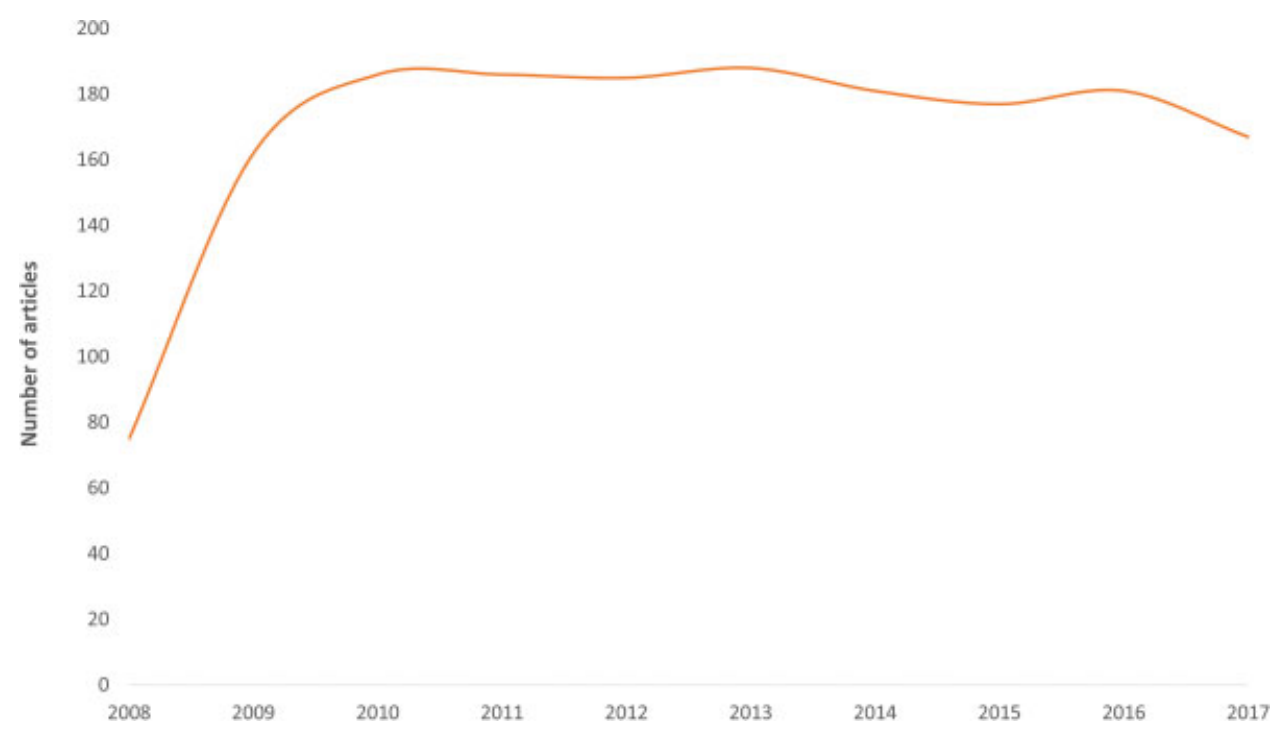

Fig. 1 Number of articles yielded per year.

such as new cannulas or less painful injections. Third and fourth most common article themes were "danger of fillers" and "celebrity use."

About $41.0 \%$ of articles listed one or more advantages, with the most common advantages being minimally invasive at $27.4 \%$, natural or subtle look at $20.0 \%$, reversibility at $12.0 \%$, and effectiveness at $12.6 \%$. Disadvantages of facial fillers were also a common theme among the news articles with $46.0 \%$ of papers listing one or more. The most common disadvantage listed was complications at $42.7 \%$ (-Fig. 2), with bruising, swelling, and nodule formation as the top three most frequently cited. (-Table 1) Examining trends in the number of news articles reporting complications ( - Fig. 3 ), a significant increase was noted from 2008 to 2017 ( $p<0.05$ ).

In addition, $46.2 \%$ of papers discussed a facial compartment or location that fillers are used for, with cheeks, lips, and eyes being the three most frequently reported sites at 22.6, 20.7, and
$12.9 \%$, respectively. Further analysis of filler brands cited by news articles showed that $63.9 \%$ of articles mentioned specific fillers by brand, with Restylane (Galderma Laboratories, L.P.) and Juvederm mentioned $28.8 \%$ of the time, followed by Radiesse (Merz North America) and Scupltra (Galderma Laboratories, L.P.) at 10.8 and $11.9 \%$, respectively. About $18.7 \%$ of news articles discussed the benefits of one filler over another, including ability to dissolve, duration of action, danger of silicone, and safety profile. About $12.4 \%$ of articles also included a physician advertisement, either directly or indirectly.

Overall, $44.7 \%$ of the articles were positive, $26.1 \%$ were negative, and 29.2\% were neutral. Examining trends in facial fillers news coverage over time revealed there was no significant difference in positive news coverage from 2008 to 2017. Although there was a simultaneous decrease in negative coverage and increase in positive coverage, this difference did not

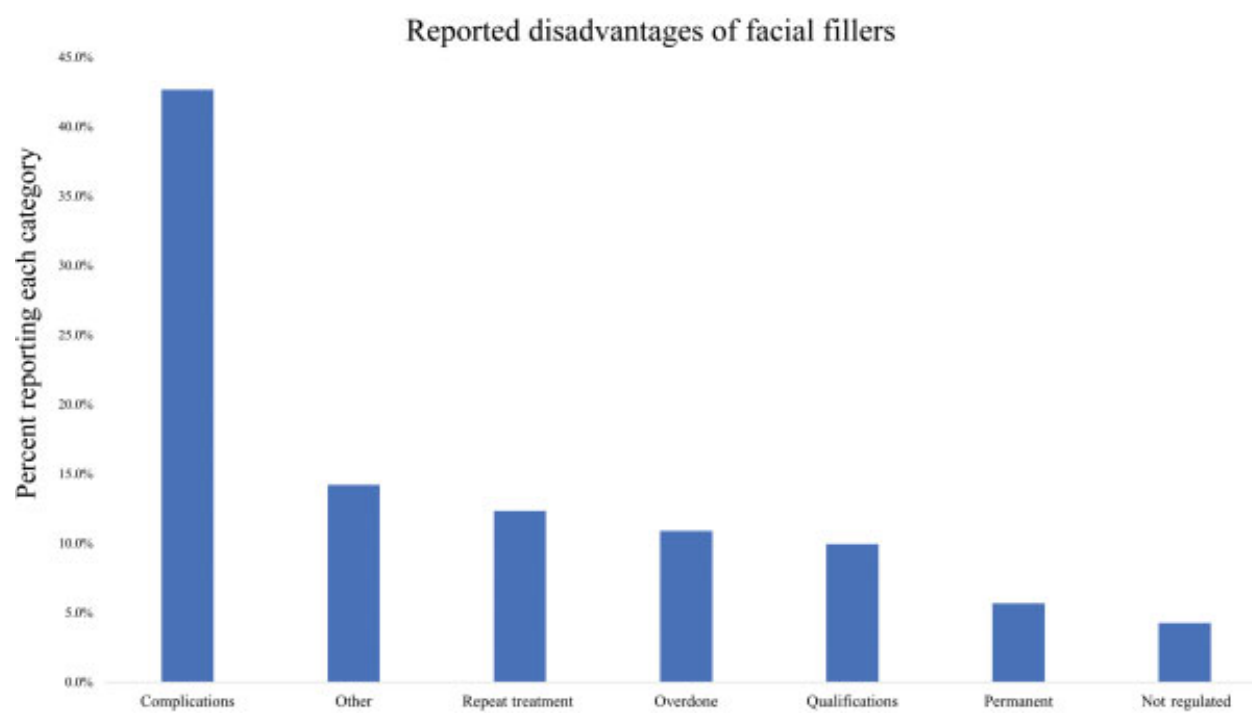

Fig. 2 Distribution of reported disadvantages of facial fillers, including complications, need for repeated treatments, injections that are overdone, concern about provider qualifications, permanency of silicone fillers, and regulation concerns, as well as others. 
Table 1 Breakdown of complications

\begin{tabular}{|l|l|l|}
\hline Complication type & Number & Percent \\
\hline Bruising & 63 & $20.1 \%$ \\
\hline Swelling & 53 & $16.9 \%$ \\
\hline Nodules & 44 & $14.1 \%$ \\
\hline Infection & 39 & $12.5 \%$ \\
\hline Allergic reaction & 29 & $9.3 \%$ \\
\hline Blindness & 20 & $6.4 \%$ \\
\hline Scar & 18 & $5.8 \%$ \\
\hline Vascular injury & 18 & $5.8 \%$ \\
\hline Nerve damage & 14 & $4.5 \%$ \\
\hline Migration & 15 & $4.8 \%$ \\
\hline Total complications & 313 & $100 \%$ \\
\hline
\end{tabular}

reach statistical significance $(p=0.12)$. Analyzing international, local, and national news coverage also revealed no significant difference in positive versus negative news coverage ( - Fig. 4). Finally, out of the news articles listing a physician specialty, plastic surgery (not including facial plastic surgery) and dermatology were the most commonly listed professions, followed by others category which included dentistry, ophthalmology, aesthetician, and finally otolaryngology and facial plastic surgery, as a distant third.

\section{Discussion}

Over the past decade, there has been a significant increase in demand for facial fillers due to their noninvasive nature and increased popularity in social media. ${ }^{12}$ The multitude of new products entering the market represents a sign of persistent demand, with Allergan alone introducing five new US Food and Drug Administration (FDA) approved products to the US market over the past decade. ${ }^{13}$ Google News is a popular resource used by the general population to stay up to date on current events. Previous analyses have demonstrated how news article coverage can have a powerful impact on shaping public opinion about screening procedures and controversial surgeries. ${ }^{10,11}$ Particularly with facial fillers, debate over the safety of materials, celebrity news, and endorsements can significantly sway patient's interests, questions, and expectations. Tabloids, blogs, and other smaller news agencies are often times sponsored, in some way, by industry sources and therefore provide a powerful tool for companies to use to shape public perception surrounding their medical treatments.

No previous study has specifically looked at how news articles have covered facial fillers over the past decade. The aim of this analysis was to categorize news sources, their authors, locations, content, and overall stance on facial fillers over the past decade. Such information can help guide physicians to better understand patient's preconceived notions and expectations of an exponentially increasing elective medical procedure.

After an initial rise and peak in the number of articles by 2010, the quantity of news articles covering facial fillers remained steady at 180 to 190 ( $\mathbf{- F i g . ~ 1 ) . ~ T h e ~ F D A ~ b e g a n ~}$ approval of HA-based fillers in 2003, with further approval of longer-lasting modified HA-based fillers, and the introduction of Poly-L-Lactic Acid, in 2009. FDA approval during this time can account for the surge of news coverage seen starting around 2008 to 2009. Although this analysis only looked at articles written in the English language, it was surprising that less than half originated from the United States, with significant coverage in Europe. Although the news articles covered a variety of topics, nearly $42.7 \%$ of articles listed a complication from facial fillers. There was also a significant increase in how often complications were reported from 2008 to 2018. Bruising was listed as the most common complication at $20.1 \%$, with more serious sequelae of vascular injury, including vision loss being reported at $\sim 12.2 \%$ $(-$ Table 1$)$. The true rate of tissue necrosis in the literature is
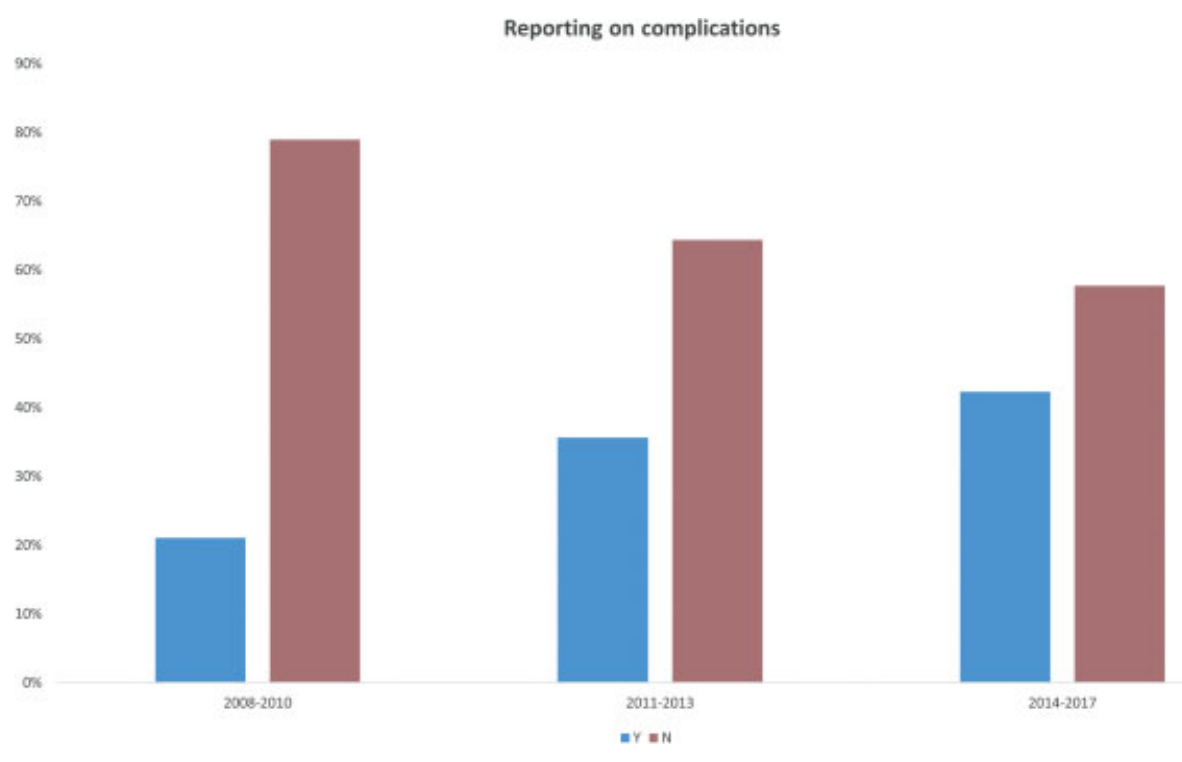

Fig. 3 Trend in news articles listing complications. 


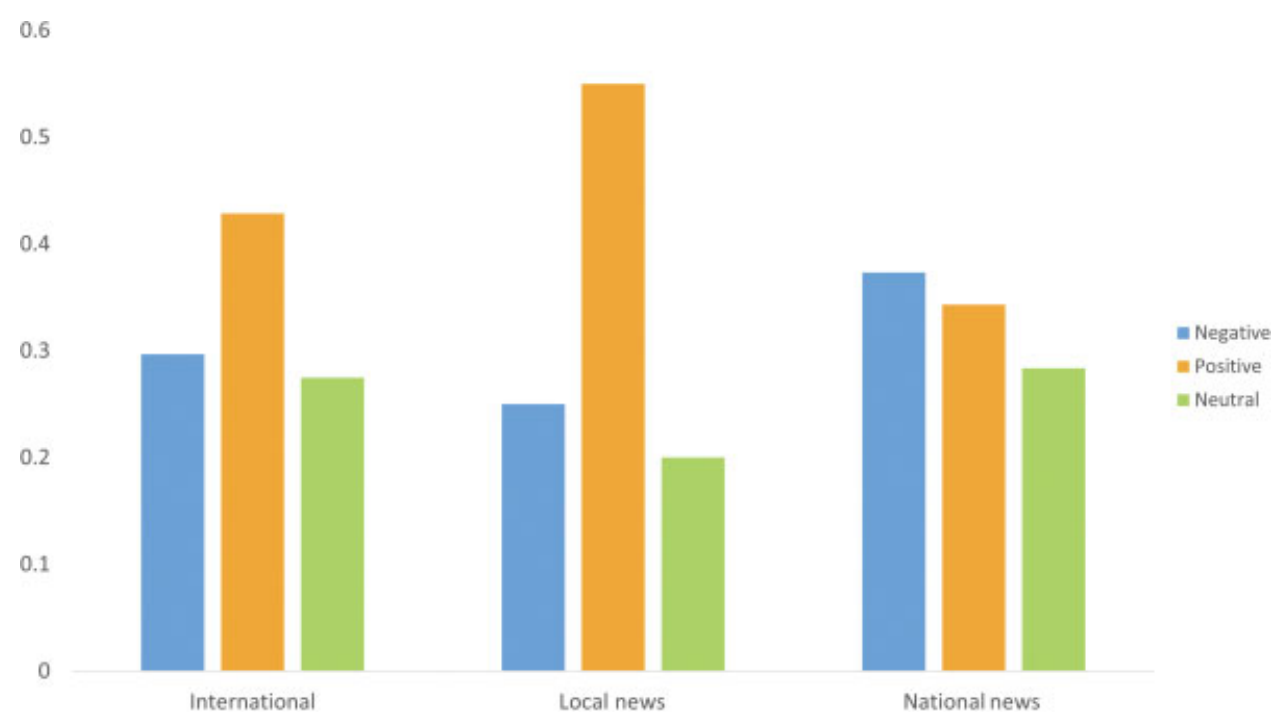

Fig. 4 Media coverage of facial fillers by article source.

variable depending on the product injected, location that it's placed, and experience of the practitioner. ${ }^{14}$ However, it is estimated to be extremely rare with an occurrence of less than $0.1 \%{ }^{15}$ The attention this complication is receiving in the news media is disproportionate to its true incidence.

In comparison, one news article which discusses adverse outcome data from Juvederm and Restylane products indicates that an average of $67 \%$ of patients will experience bruising after injection. ${ }^{16}$ This suggests that bruising is the most common complication of filler injections. Yet in the present study, bruising was reported in only $20.1 \%$ of articles which mentioned adverse effects of facial filler injections. In this regard, sensationalism in news articles on facial fillers may be skewed toward reporting the most severe complications with higher frequency. Although the motivation for overreporting of severe complications is likely to produce attention-grabbing headlines, certain publications such as tabloids and blogs may be more subject to individual bias than larger organizations. Our results indicate a larger percent of local news agencies produced positive news articles on facial fillers, as compared with national or international news agencies. Although the difference was not statistically significant, this study may have been underpowered to elicit signs of potential publication bias.

Previous studies have shown an intricate relationship between changes in healthcare policy and news article opinion on such policy. Examples include appropriate age for screening mammography ${ }^{11}$ and media coverage after FDA's opinion on surgical mesh for pelvic organ prolapse. ${ }^{10}$ Since the popularization of facial fillers, there has been an ongoing debate regarding their safety for off-label use, longterm effects, and who should be qualified to perform the procedures. This is particularly relevant in the United Kingdom where no qualifications are needed for an individual to perform these injections. ${ }^{17}$ In addition, due to fillers being classified as nonpharmaceutical, there are over 100 different brands of fillers available in the United Kingdom, including "do it yourself" home kits. In the United States, there is greater regulation with the FDA currently providing a comprehensive list of approved dermal fillers, warning against unapproved uses and the side effects of facial fillers. The administration further recommends seeking licensed healthcare professionals, who preferably have experience in the field of dermatology or plastic surgery.

However, the organization fails to define what constitutes a "licensed healthcare professional." The American Medical Association defines this term as "an individual who is qualified by education, training, licensure/regulation (when applicable) and facility privileging (when applicable) who performs a professional service within his/her scope of practice and independently reports that professional service." ${ }^{18}$ This includes nurse practitioner, certified nurse specialist, physician assistant, certified nurse mid-wife, certified registered nurse anesthetist, and even clinical social worker, or physical therapist. This list includes a wide array of healthcare professionals, among which many are not considered adequately trained to administer facial filler injections.

Previous studies have shown litigations in plastic surgery are commonly due to a practitioner's lack of expertise, lack of informed consent, and poor cosmetic outcome. ${ }^{19,20}$ With the mean monetary award totaling $\$ 242,000$ and greater than $50 \%$ of patients sustaining permanent injury, ${ }^{14}$ further regulation of what defines "qualified" is paramount to our healthcare system. Particularly with the exponential increase in facial filler demand and its interdependence with popular media, future news coverage both locally and internationally can play a vital role in public perception and government policies.

While this study underscores important trends and news media coverage of facial fillers, it does have several limitations. First, Google News was chosen since it is a convenient means to sample news articles with the hope that this powerful search engine would represent online news coverage of facial fillers as a whole. However, as no single resource can provide, Google News does not serve as an all-inclusive list of news articles produced about a given topic. Nonetheless, this resource has been invaluable in prior analyses examining news coverage of 
different medical topics and appeared as an appropriate resource for assessing media coverage of facial fillers. ${ }^{8,10,11}$ Although all attempts were made to create an objective review process (e.g., using three independent evaluators), inherent biases could not be entirely ruled out. While most major international journals provide articles in English, limiting the search to the English language would invariably reduce coverage of non-English speaking countries. This likely explains why $90 \%$ of international articles originated in Great Britain. Finally, there is variability in the number of articles yielded between various media sources for several our analyses. Therefore, readers should use caution when drawing conclusions based on data from the lower-yield media sources. Nonetheless, this analysis provides a comprehensive review of how news media has been covering an ever-evolving field of facial fillers over the past decade.

\section{Conclusion}

News media sources generally provide positive coverage of facial fillers. There is a substantial focus on rare complications in the media, and may be the source of a potential misrepresentation, altering public perception. Though lack of government regulation has been a popular theme discussed in medical news topics, few policy changes have been made, particularly abroad. Demand for facial fillers, and subsequently products available and practitioners providing them will continue into the foreseeable future. Further venues to help guide public perception and policy changes may be warranted.

\section{Conflicts of Interest}

None.

\section{References}

1 American Society of Plastic Surgeons. Plastic surgery statistics report. American Society of Plastic Surgeons National Clearinghouse; 2018

2 Bass LS. Injectable filler techniques for facial rejuvenation, volumization, and augmentation. Facial Plast Surg Clin North Am 2015;23(04):479-488
3 Monheit GD, Coleman KM. Hyaluronic acid fillers. Dermatol Ther 2006;19(03):141-150

4 Philipp-Dormston WG. [Hyaluronic acid fillers in dermatology]. Hautarzt 2018;69(06):491-509

5 Kontis TC. Contemporary review of injectable facial fillers. JAMA Facial Plast Surg 2013;15(01):58-64

6 Ward B, Ward M, Paskhover B. Google trends as a resource for informing plastic surgery marketing decisions. Aesthetic Plast Surg 2018;42(02):598-602

7 Fox S, Rainie L. Vital decisions: how internet users decide what information to trust when they or their loved ones are sick. Pew Internet \& American Life Project; 2002

8 Dasgupta N, Mandl KD, Brownstein JS. Breaking the news or fueling the epidemic? Temporal association between news media report volume and opioid-related mortality. PLoS One 2009;4(11):e7758

9 About Google News. Google; 2013

10 Stone BV, Forde JC, Levit VB, Lee RK, Te AE, Chughtai B. Trends in internet search activity, media coverage, and patient-centered health information after the FDA safety communications on surgical mesh for pelvic organ prolapse. Int Urogynecol J Pelvic Floor Dysfunct 2016;27(11):1761-1766

11 Young Lin LL, Rosenkrantz AB. The U.S. online news coverage of mammography based on a Google News search. Acad Radiol 2017;24(12):1612-1615

12 Top 5 cosmetic minimally-invasive procedures. American Society of Plastic Surgeons; 2016

13 Dermal fillers approved by the center for devices and radiological health. The United States Food \& Drug Administration; 2018

14 Rayess HM, Svider PF, Hanba C, et al. A cross-sectional analysis of adverse events and litigation for injectable fillers. JAMA Facial Plast Surg 2018;20(03):207-214

15 Ferneini EM, Ferneini AM. An overview of vascular adverse events associated with facial soft tissue fillers: recognition, prevention, and treatment. J Oral Maxillofac Surg 2016;74(08):1630-1636

16 Alster T Dr. Alster: Bruising Affects 67\% of Dermal Filler Patients. http://www.cearna.com/blog/dr-alster-bruising-affects-67-ofdermal-filler-patients/. Accessed 8/21/18

17 The National Health Service (NHS). Review of qualifications required for delivery of non-surgical cosmetic interventions. The National Health Service Constitution; 2014

18 Joette Derricks M. Define a Qualified Healthcare Professional. The American Academy of Professional Coders; 2015

19 Kandinov A, Mutchnick S, Nangia V, et al. Analysis of factors associated with rhytidectomy malpractice litigation cases. JAMA Facial Plast Surg 2017;19(04):255-259

20 Mehta S, Farhadi J, Atrey A. A review of litigation in plastic surgery in England. Lessons learned. J Plast Reconstr Aesthet Surg 2010; 63(10):1747-1748 